Bar-On, A. 54

Chelas, E.-N. 20

Ferman, S. 8, 54

Fernandes, F.D. 8, 78

Gillon, G. 5, 6, 8

Hus, Y. 8, 27

Hyter, Y. 6, 8, 20

Kaloudi, V. 20

Kaplan, D. 67

Kishon-Rabin, L. 67

Kivrakidou, E. 20

Minaidou, D. 38
Paparizos, K. 20

Patael, S. 67

Petinou, K. 8, 38

Segal, O. 8, 67

Sun, I.Y.I. 78

Tumanova, T. 8

van Bysterveldt, A.K. 43

Varanda, C.A. 78

Vogindroukas, I. 8, 20

Westby, C. 8

Westerveld, M. 6, 8, 43

\title{
Subject Index Vol. 69, No. 1-2, 2017
}

Adolescents 8

- with autistic spectrum disorder 67

Assessment 8, 20

Autism 8, 20, 43, 78

- issues 27

- spectrum disorder $8,38,54$

Children 8

Cognition 78

Diagnosis 8

Down syndrome 43

Early intervention 38

Executive function 78

Eye gaze 38

Graphemic-phonemic decoding 54

Home literacy environment 43

Intervention 8
Joint attention 38

Language 78

Morpho-orthographic identification 54

Narrow focus 67

Neurobiology 38

Pragmatics 20, 78

Preschoolers 43

Prosody 67

Pseudowords 54

Reading 54

Resource toolkit 27

Shared book reading 43

Social communication 20

Speech-language pathologists 8,27

Survey 8 